\title{
Vegetative development and berry growth in relation to heat accumulation in Sangiovese vines subjected to double pruning at three different times
}

\author{
Vania Lanari ${ }^{1, *}$, Tania Lattanzi ${ }^{1}$, Bruno Di Lena ${ }^{2}$, Alberto Palliotti ${ }^{3}$, and Oriana Silvestroni ${ }^{1}$ \\ 1 Dipartimento di scienze Agrarie, Ambientali ed Alimentari, D3A, Via Brecce Bianche 10, 60131 Ancona, Italy \\ ${ }^{2}$ Regione Abruzzo, Ufficio coordinamento servizi vivaistici e agrometeorologici, Scerni (Chieti), Italy \\ ${ }^{3}$ Dipartimento di Scienze Agrarie, Alimentari e Ambientali, Università di Perugia, 06121, Perugia, Italy
}

\begin{abstract}
The double pruning on Sangiovese based on pre-pruning and finishing in March, April and May applied over three years, induced a postponement of phenological phases, with repercussions on the vine vegetative and berry growth trends, with greater effects, according to the delay in the time of hand follow up during the season. The phenological development, the canopy and berry growth of Sangiovese, subjected to the three different finishing dates for the final pruning, were recorded during three years (2014-2016) and related to day of the year (DOY) and seasonal heat accumulation (growing degree days, GDD). A sigmoid growth model with high coefficient determination (R2 between 0.96 and 0.99 ), described shoot elongation and herbaceous berry growth, showing a temporal and thermal shift according to the finishing times. Despite the chronological delay, the vines revealed similar thermal necessities. Leaf area evolution of April and May finished vines showed 2 steps: a slow initial development followed by a rapid growth phase (91$97 \mathrm{~cm} 2 / \mathrm{GDD}$ ) beginning when shoots reached 7-10 leaves. GDD allowed to obtain simple models of vegetative development and berry growth of Sangiovese vines based on thermal evolution.
\end{abstract}

\section{Introduction}

Thermal increase, due to the climate change, which has caused an advance of grapevine phenology and accelerated grape ripening, has induced some changes of cultivation techniques [1]. Among these, techniques aiming to delay phenology in the form of later winter pruning $[2,3]$ or double pruning in different times $[4,5]$ have been imposed on vines with positive effects on yield and grape composition.

The double pruning of cordon trained grapevines, based on pre-pruning during the winter period and a delayed hand finishing of pre-pruned canes, is spreading also as a technique to counteract the rapid accumulation of sugars or low titratable acidity in grapes, exploiting the possibilities offered by mechanization and the grapevine acrotony.

As reported in two studies on Sangiovese [4, 5], the finishing of winter pruning after the bud break of distal buds in pre-pruned canes induces a shift of the main phenological phases and limits yield capacity. With the high potential to reduce yield, this technique is used on high-yielding vines [6], replacing the bunch thinning treatment without the additional costs of thinning interventions.

It is also well known that the grapevine cycle is strongly influenced by the thermal seasonal trend, as shown by the close correlation between bioclimatic indexes and phenological phases, including the harvest data [7].

Grapevines receiving their finishing treatment later in the season experience different environmental conditions during shoot and berry growth. The forecasting model of the degree-days was applied to estimate bud burst, vegetative development and berry growth on Sangiovese vines subjected to postponed finishing of winter pruning.

\section{Materials and Methods}

Over the three years (2014-2016), cordon trained and vertical shoot positioned Sangiovese vines were prepruned during the dormant period shortening canes to 7 8 nodes each. The vines were planted in 2004 with certified virus-free cuttings of cv. Sangiovese (clone R24) grafted onto Kober 5BB rootstock, oriented north north east to south south west and planted at $1.20 \mathrm{~m}$ vine spacing and $2.75 \mathrm{~m}$ row spacing (3030 vines/ha). Vines were not irrigated. Finishing to spur cordon (Fig. 1) occurred in March in control vines (CK), April in vines that showed 3-4 extended leaves from distal buds (DF3) and May in vines that showed 7-8 extended leaves (DF7). During the three years of the trial, the phenology of grapevines was monitored according to the classification from stage $\mathrm{BBCH0}$ (dormant bud) to stage BBCH-19 (shoot with unfolded leaves). Shoot elongation was measured as well as total leaf area and 
berry growth. Daily meteorological data were taken from the Regional Hydrographic Service (Protezione Civile della Regione Marche). The daily mean temperature values over the three years were used to calculate growing degree days (GDD, base $10^{\circ} \mathrm{C}$ ) and the heat accumulation from January $1^{\text {st }}$ to the main phenological phases. The GDD model was used to describe vegetative development (shoot elongation and leaf area formation) and berry growth. The maximum shoot length was determined by topping $0.3-0.4 \mathrm{~m}$ above the upper foliage wire.

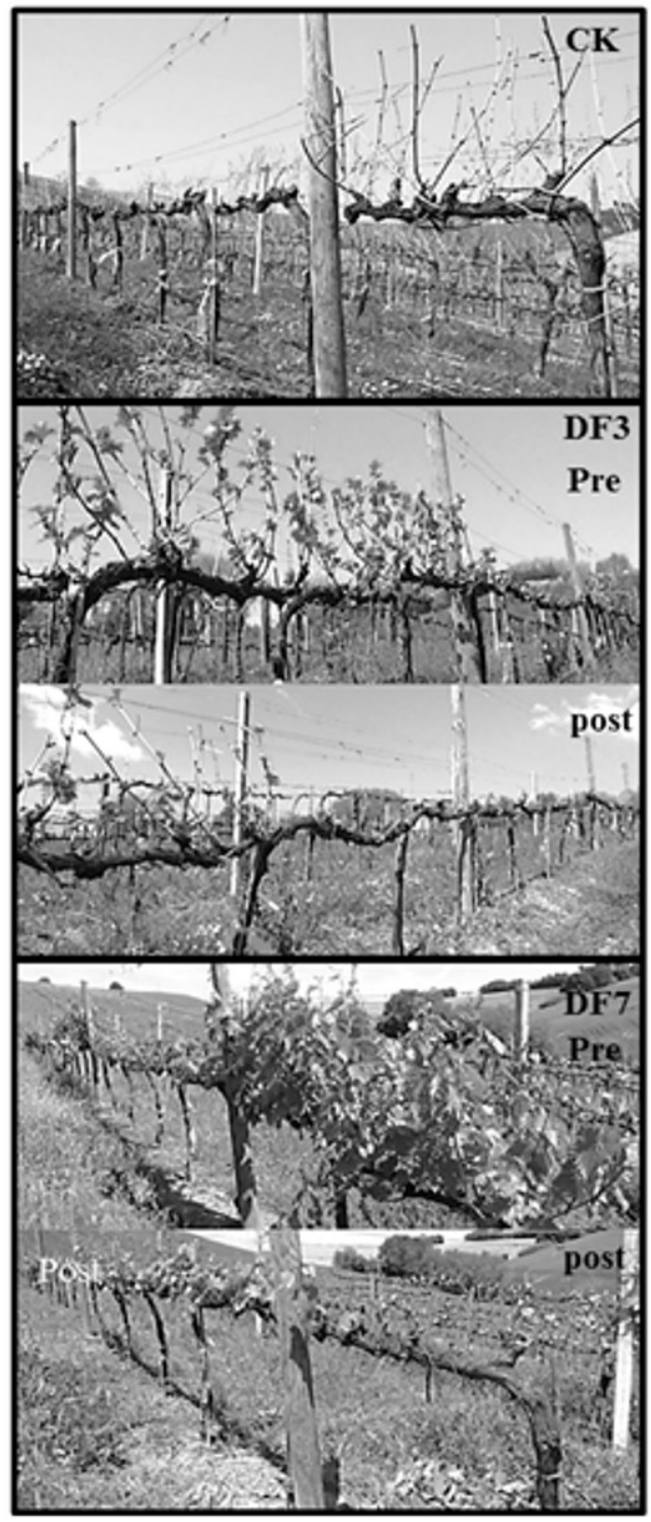

Fig. 1. (CK) Control grapevine pre-pruned and finished in March; (DF3) grapevines pre-pruned in March (pre) and finished in April; (DF7) grapevines pre-pruned in March (pre) and finished in May.

\section{Results and Discussions}

From 2014 to 2016 , CK vines reached $50 \%$ of buds in phenological phase $\mathrm{BBCH}-7$ (green shoot tips just visible) and $\mathrm{BBCH}-11$ (first leaf unfolded and spread away from shoot), 98 and 105 days from January $1^{\text {st }}$.

The corresponding heat accumulation was 95 and 141 GDD, respectively. Shoot emission from distal buds of the pre-pruned canes in the DF3 and DF7 vines required similar GDD and exerted an acrotonic behaviour (correlative inhibition) along the cane: the basal buds did not break, as shown in previous studies $[4,5]$. The DF3 and DF7 vines, reached bud break in basal buds later, reaching $50 \%$ of buds in phenological phase BBCH-7 on DOY 124 and 138 respectively. The phenological phase BBCH-11 was reached on DOY 138 and 146 in DF3 and DF7, which required increased heat accumulations, compared to $\mathrm{CK}$ vines, according to the delay in finishing intervention time (Tab. 1).

The longer period shown by the DF vines in the basal bud burst is due, especially, to the lapse of time from January to the finishing intervention, which is obviously longer in the late-finished vines with higher thermal accumulations than the control.

The postponement of the finishing intervention to 1 or 3 weeks after the budburst of distal nodes caused a delay of 25-40 days in the budburst of basal buds. While $\mathrm{CK}$ vines required 30 days after the finishing to reach basal budburst, DF3 and DF7 required only 15 and 13 days respectively. Delayed finishing of pruning shifted basal bud development of DF vines into a warmer period, thus heat accumulated between finishing and basal budburst (phenological phase BBCH-7) increased to 76 and 95 GDD in DF3 and DF7, +22 and + 41 GDD, respectively, as compared to $\mathrm{CK}$ vines. Shoot emission (phenological phase BBCH-11) followed a similar pattern and the heat requirement from finishing increased from the 90 GDD of $\mathrm{C}$ vines to 152-156 GDD of DF vines (Tab. 1).

Table 1. Days and heat accumulation (GDD) from January 1st to basal dud development (Jan-basal bud dev.); from January 1 st to finishing of winter pruning (Jan- Fin. WP); and from finishing of winter pruning to basal bud development (Fin WPbasal bud dev.). $\mathrm{PP}=$ Phenological Phase. $\mathrm{BBCH}-7=$ budburst;

$\mathrm{BBCH}-11=$ shoot emission. Means \pm S.D are shown

\begin{tabular}{|c|c|c|c|c|c|}
\hline GDD & & PP & CK & DF3 & DF7 \\
\hline \multirow{4}{*}{$\begin{array}{c}\text { Jan-basal } \\
\text { bud dev. }\end{array}$} & \multirow{2}{*}{ Days } & ВBCH-7 & $98 \pm 4$ & $124 \pm 4$ & $138 \pm 5$ \\
\hline & & BBCH-11 & $105 \pm 6$ & $134 \pm 3$ & $146 \pm 5$ \\
\hline & \multirow{2}{*}{ GDD } & BBCH-7 & $95 \pm 24$ & $226 \pm 25$ & $327 \pm 19$ \\
\hline & & BBCH-11 & $141 \pm 24$ & $302 \pm 37$ & $388 \pm 33$ \\
\hline \multirow{2}{*}{$\begin{array}{c}\text { Jan- } \\
\text { Fin. WP }\end{array}$} & Days & & $69 \pm 12$ & $110 \pm 6$ & $126 \pm 1$ \\
\hline & GDD & & $41 \pm 24$ & $150 \pm 20$ & $232 \pm 17$ \\
\hline \multirow{4}{*}{$\begin{array}{c}\text { Fin. WP- } \\
\text { basal bud } \\
\text { dev. }\end{array}$} & \multirow{2}{*}{ Days } & BBCH-7 & $30 \pm 12$ & $15 \pm 5$ & $13 \pm 5$ \\
\hline & & BBCH-11 & $37 \pm 12$ & $26 \pm 4$ & $21 \pm 5$ \\
\hline & \multirow{2}{*}{ GDD } & BBCH-7 & $54 \pm 4$ & $76 \pm 6$ & $95 \pm 23$ \\
\hline & & BBCH-11 & $90 \pm 24$ & $152 \pm 23$ & $156 \pm 50$ \\
\hline
\end{tabular}

Logistic growth curve was used to model shoot elongation in relation to DOY and GDD (Fig. 2). The model revealed high determination coefficients values $\left(\mathrm{R}^{2}\right)$ ranged from 0.95 to 0.97 in DOY relationship and 
equal to 0.98 in GDD model. The logistic growth curve is characterized by a slow initial phase, followed by a rapid growth of shoot, and a slow final phase, equivalent to the maximum shoot length, determined with the topping intervention.

Over the three years, the delay in basal bud burst in DF vines was reflected in a temporal and thermal delay of the shoots' elongation as shown by the shifting of the point of maximum growth rate that corresponds to the
$50 \%$ of the final shoot length, reached by $\mathrm{C}$ vines on DOY 142 with heat accumulation of 361 GDD

The DF3 and DF7 vines reached the point of maximum growth rate on DOY 158 and 171 respectively, corresponding to heat accumulations values of 533 and 688 GDD respectively. However, all vines reached a final length of $1.4 \mathrm{~m}$ in 130 days (Fig. 1,A) with similar thermal necessities-between 400 and 462 GDD (Fig.2, B).

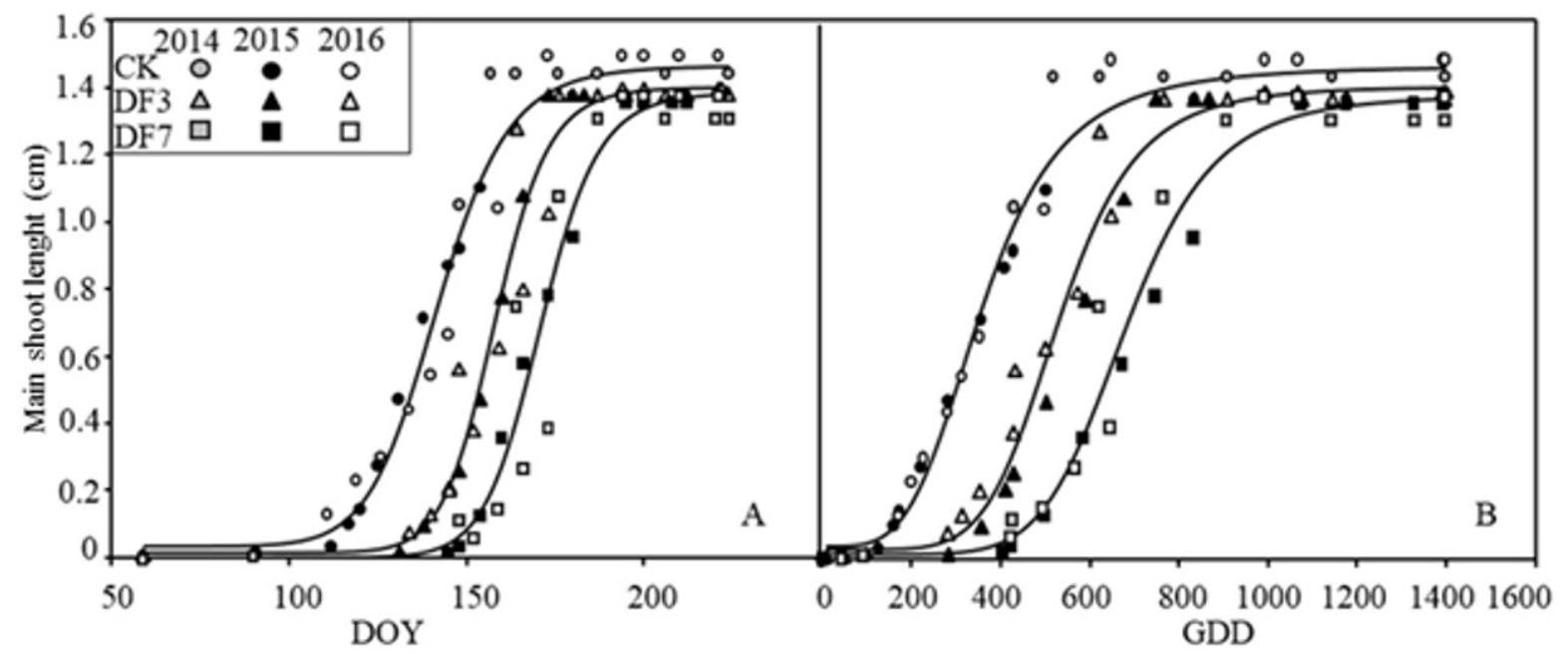

Fig. 2. Relationships between main shoot length (m) and (A) day of the year (DOY); and (B) growing degree days (GDD), over the three years 2014-2016

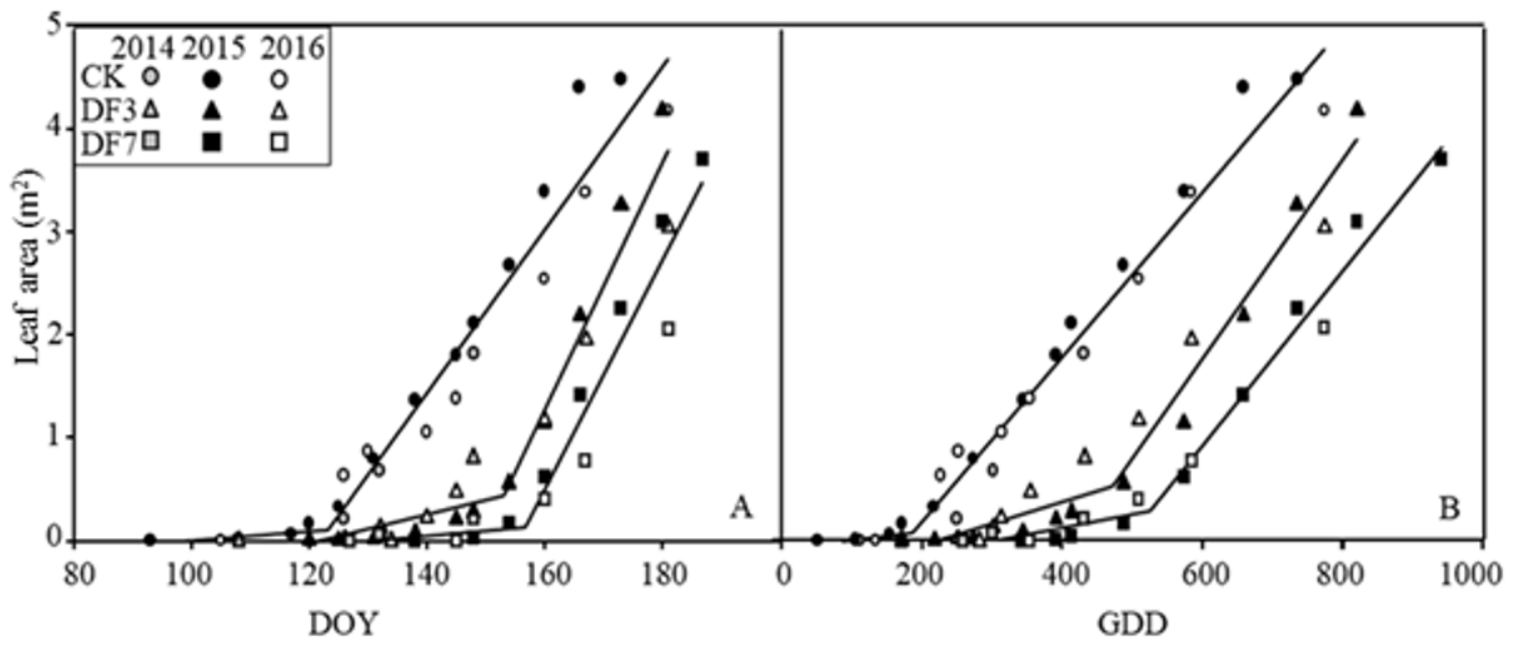

Fig. 3. Relationships between leaf area per vine (m2) and (A) day of the year (DOY); and (B) growing degree days (GDD), over the three years 2014-2016 
The rapid growth phase of the logistic curve can also be described by using simple linear regression, making it easy to compare among treatments. Shoot elongation rates in DF vines ranged between 3.5-3.6 $\mathrm{cm}$ per day versus $2.9 \mathrm{~cm} /$ day of $\mathrm{CK}$ vines. When shoot growth was related to GDD model, the difference among treatments dramatically decreased and shoot elongation rate of Sangiovese vines ranged around 0.3 $\mathrm{cm} / \mathrm{GDD}$, irrespective of treatments (data not shown).

The delay in finishing pruning, and the consequent delay in shoot elongation, induced a postponement of leaf area evolution in the DF vines. The relationships between leaf area per vine and DOY or GDD models were described by piecewise functions: a slow initial canopy development occurred until the formation of 710 leaves per shoot and was followed by a phase of rapid growth. The piecewise functions showed high $\mathrm{R}^{2}$ values ranging from 0.88 to 0.90 in DOY relationship and around 0.94 with GDD model (Fig. 3, A and B). The rapid leaf area evolution in DF vines ranged from1198-1109 $\mathrm{cm}^{2}$ per day versus $791 \mathrm{~cm}^{2} /$ DOY of CK vines (Fig. 3, A). When leaf area was related to GDD model, the difference among treatments reduced and leaf area evolution of vines ranged from 91 $\mathrm{cm}^{2} / \mathrm{DD}$ in DF3 and $97 \mathrm{~cm}^{2} / \mathrm{DD}$ in DF7 vines vs 80 $\mathrm{cm}^{2} / \mathrm{DD}$ in $\mathrm{CK}$ vines (Fig. 3, B).

Initially, the growth of leaves is slow as it is mainly sustained by the remobilization of reserves stored in the vine organs during the previous summer [8]. When the leaves reached about one-third of final size, they began exerting high photosynthetic rates, acting as an active source of carbohydrates for sinks organs [9], therefore, also, able to sustain the growth alone.

Over the three years, the delay in basal budburst in DF vines was reflected in a temporal and thermal delay of the subsequent phenological phases. The time between bud burst and bloom was, on average, 50 days for CK vines, 35 and 30 days for DF3 and DF7 respectively, all requiring similar heat accumulation. The shortest lapse of time between basal budburst and bloom in the DF vines was compensated by high daily mean temperatures. From bloom to fruit-set, vines required about 10 days with heat accumulation increase according to the delay of finishing of the winter pruning times. The lapse of time and GDD accumulation from fruit-set to veraison were similar between vines (Tab. 2).

The delay in the bloom and fruit set dates in DF vines was reflected in a temporal and thermal shift of the berry development according to the time of the winter pruning. The typical double sigmoid model described berry growth in relation to DOY and GDD, and logistic growth curve was used to model herbaceous berry growth. The model revealed high $\mathrm{R}^{2}$ values and the herbaceous growth is characterized by a slow initial phase, followed by a rapid berry growth attaining about $50 \%$ (around $1.5 \mathrm{~g}$ ) of the final berry weight. The veraison and berry ripening phase was characterized by variability of data, due mainly to the seasonal trend revealing its influence on berry development, which occurred in the hottest period, well-evident in the 2015 season (Fig. 4) characterized by summer drought [1].

Table 2. Days and heat accumulation (GDD) between the main phenological phases. Means \pm S.D are shown.

\begin{tabular}{|c|c|c|c|c|}
\hline & & CK & DF3 & DF7 \\
\hline \multirow{2}{*}{$\begin{array}{c}\text { bud burst } \\
\text { to bloom }\end{array}$} & Days & $50 \pm 6$ & $35 \pm 6$ & $30 \pm 1$ \\
\cline { 2 - 5 } & GDD & $319 \pm 8$ & $310 \pm 51$ & $363 \pm 19$ \\
\hline \multirow{2}{*}{$\begin{array}{c}\text { bloom to } \\
\text { fruit-set }\end{array}$} & Days & $9 \pm 2$ & $9 \pm 1$ & $10 \pm 3$ \\
\cline { 2 - 5 } & GDD & $88 \pm 25$ & $111 \pm 10$ & $132 \pm 64$ \\
\hline \multirow{2}{*}{$\begin{array}{c}\text { fruit-set to } \\
\text { veraison }\end{array}$} & Days & $56 \pm 3$ & $56 \pm 7$ & $50 \pm 8$ \\
\cline { 2 - 5 } & GDD & $796 \pm 42$ & $801 \pm 40$ & $752 \pm 125$ \\
\hline
\end{tabular}

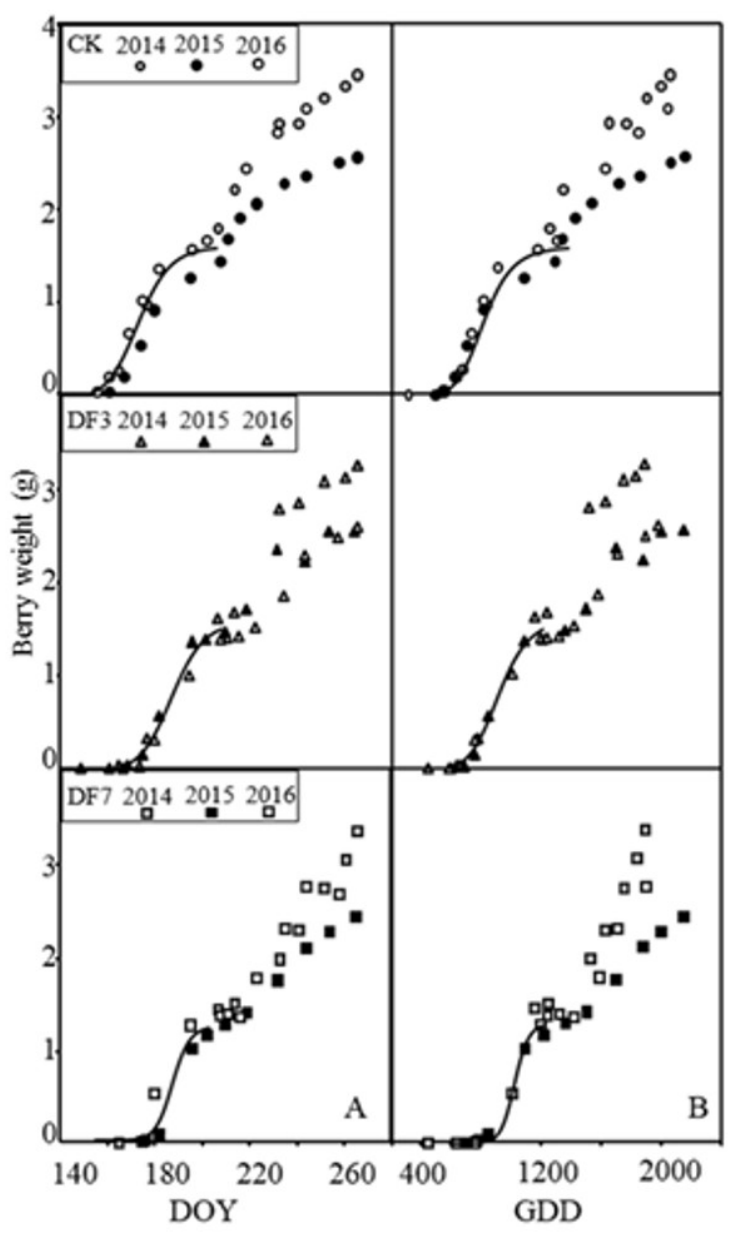

Fig. 4. Relationships between berry weight (g) and (A) day of the year (DOY); and (B) growing degree days (GDD), over the three years 2014-2016.

\section{Conclusions}

Double pruning based on pre-pruning during the dormant period and finishing of winter pruning in different times in the season, over the three years, induced a temporal and thermal shift of phenological phases. However, the GDD model showed lower 
variability of data than DOY. Despite the chronologic delay in the main phenological phases, the vines revealed similar heat requirements among them.

The study of the vegetative development and berry herbaceous growth based on seasonal thermal courses (GDD) provided vegetative and productive growth models that can be used for the vine phenological description, allowing one to program the cultural techniques on the basis of thermal data, reducing, in this way, the phenological observation in field.

\section{References}

1. 1. A. Palliotti, S. Tombesi, O. Silvestroni, V. Lanari, M. Gatti, S. Poni. Sci Hortic,. 17,:43-54 (2014)

2. T. Frioni, S. Tombesi, O. Silvestroni, V. Lanari, A. Bellincontro, P. Sabbatini, Am. J. of Enol. and Vitic, 67, 419-425 (2016)

3. M. Gatti, F.J. Pirez, F.J., Chiari, S. Tombesi, A. Palliotti, M.C. Merli, S. Poni, Front. in Plant Sciences, 7, 659 (2016).

4. O. Silvestroni, V. Lanari, T. Lattanzi, A. Palliotti. Austr. J. Grape and Wine Res., (to be publisher). DOI: 10.1111/ajgw.12361 (2018)

5. A. Palliotti, T. Frioni, S. Tombesi, P. Sabbatini, J.G. Cruz-Castillo, V. Lanari, O. Silvestroni, M. Gatti, and S. Poni, Am. J. of Enol. and Vitic, 68, 412-421 (2017).

6. O. Silvestroni, V. Lanari, T. Lattanzi, A. Palliotti, P. Sabbatini, Am. J. of Enol. and Vitic, 67, 407418 (2016).

7. B. Di Lena, O. Silvestroni, V. Lanari, A. Palliotti, Theore. and App. Climat, 1-11 (2018).

8. C. Zapata, E. Deléens, S. Chaillou, C. and Magné. J. of Plant Phys, 16, 1031-1040 (2004)

P.R. Petrie, M.C.T. Trought, and G.S. Howell, Vitis, 39, 31-36 (2000). 